\title{
Intravenous Carnitine Administration in Addition to Parenteral Nutrition With Lipid Emulsion May Decrease the Inflammatory Reaction in Postoperative Surgical Patients
}

\author{
Yu Koyama ${ }^{\text {a, d }}$, Kazuki Moro ${ }^{\mathrm{b}}$, Masato Nakano ${ }^{\mathrm{b}}$, Kohei Miura ${ }^{\mathrm{b}}$, Masayuki Nagahashi ${ }^{\mathrm{b}}$, \\ Shin-ichi Kosugi ${ }^{\mathrm{c}}$, Junko Tsuchida ${ }^{\mathrm{b}}$, Mayuko Ikarashi ${ }^{\mathrm{b}}$, Masato Nakajima ${ }^{\mathrm{b}}$, \\ Hiroshi Ichikawa $^{\mathrm{b}}$, Takaaki Hanyu ${ }^{\mathrm{b}}$, Yoshifumi Shimada ${ }^{\mathrm{b}}$, Jun Sakata ${ }^{\mathrm{b}}$, \\ Hitoshi Kameyama ${ }^{\text {, }}$ Takashi Kobayashi ${ }^{b}$, Toshifumi Wakai ${ }^{b}$
}

\begin{abstract}
Background: A prospective randomized study was performed to investigate the validity of intravenous carnitine administration during postoperative parenteral nutrition $(\mathrm{PN})$ with lipid emulsion.

Methods: Patients undergoing surgery for gastric or colorectal cancer were enrolled in the study and were randomly divided into two groups ( $\mathrm{n}=8$ in each group): 1 ) group $\mathrm{L}$, who received a peripheral PN (PPN) solution of 7.5\% glucose, 30\% amino acid, and 20\% lipid emulsion; and 2) group LC, who received the same PPN solution, as well as carnitine intravenously. PPN was performed from postoperative day (POD) 1 to POD4. Clinical and laboratory parameters were compared between the two groups; statistical significance was set at $\mathrm{P}<0.05$.
\end{abstract}

Results: Serum carnitine concentrations were significantly higher in group LC on POD3 $(\mathrm{P}<0.01)$ and POD7 $(\mathrm{P}=0.01)$. Postoperative changes in laboratory parameters and morbidity were comparable between the two groups. However, the decrease in C-reactive protein from POD3 to POD7 was significantly greater in group LC than in group L $(\mathrm{P}=0.011)$.

Conclusion: The results show that intravenous carnitine administration in addition to $\mathrm{PN}$ is safe and may be beneficial for recovery from postoperative inflammatory reactions.

Keywords: Carnitine; Parenteral nutrition; C-reactive protein; Lipid

Manuscript submitted July 27, 2017, accepted August 9, 2017

a Department of Nursing, Niigata University Graduate School of Health Sciences, 2-746 Asahimachi, Niigata, Niigata 951-8518, Japan

${ }^{b}$ Division of Digestive and General Surgery, Niigata University Graduate School of Medical and Dental Sciences, Niigata, Japan

'Department of Digestive and General Surgery, Uonuma Institute of Community Medicine, Niigata University Medical and Dental Hospital, 4132 Urasa, Minami-Uonuma, Niigata, Japan

${ }^{\mathrm{d} C}$ Corresponding Author: Yu Koyama, Department of Nursing, Niigata University Graduate School of Health Sciences, 2-746 Asahimachi, Niigata, Niigata 951-8518, Japan. Email: yukmy@clg.niigata-u.ac.jp

doi: https://doi.org/10.14740/jocmr3113w emulsion; Inflammatory reaction; n-6 polyunsaturated fatty acid

\section{Introduction}

Carnitine, an important nutrient, has an essential role in longchain fatty acid (LCFA) transport into the mitochondrial matrix for $\beta$-oxidation $[1,2]$. Carnitine is obtained from dietary sources [3] or synthesized from methionine and lysine endogenously [1]; approximately three-quarters of carnitine in the human body is obtained from the diet, with the remaining onequarter synthesized in the liver, kidney, brain, and placenta [4]. Generally, carnitine production in the body is sufficient; however, there are some situations in which exogenous carnitine supplementation is necessary to avoid carnitine deficiency, including metabolic disorders, renal failure, liver failure, or under parenteral nutrition (PN) [5].

PN is a vital therapeutic modality indicated for a range of conditions in neonates through to adults [6]. For example, PN is often administrated after major abdominal surgery because of limitations in enteral nutrition in the early postoperative stage $[7,8]$. Intravenous lipid emulsions are an integral part of PN [9], and the infusion of lipid emulsions allows for the supply of high energy as an iso-osmolar solution, as well as meeting requirements for essential fatty acids [10]. Therefore, to meet nutritional needs, it is useful to add a lipid emulsion including LCFA to PN regimens.

Intravenous carnitine supplementation of $\mathrm{PN}$ with lipid emulsions has been performed particularly in the neonate population to improve nutritional status or clinical outcome [11-13], but the validity of intravenous carnitine supplementation of PN in addition to lipid emulsion remains contentious. Some studies have shown no significant benefit of intravenous carnitine supplementation in terms of improving nutritional status or clinical outcome [14-16], whereas others have reported positive effects on metabolic disturbances or nutritional status $[11,17]$.

Conversely, the efficacy of intravenous carnitine supplementation of PN with lipid emulsion in adults in a starved condition after surgical stress has not yet been verified. Thus, in the present study, we prospectively investigated the validity of 
carnitine supplementation during postoperative PN with lipid emulsion.

\section{Materials and Methods}

\section{Patients enrollment}

This was a prospective, randomized study conducted by a single institution (Division of Digestive and General Surgery, Niigata University Hospital). The ethical approval of the present study (\#2015-1925) was obtained from Institutional Review Board for Clinical Research. Patients who received surgery for gastric or colorectal cancer at Niigata University Hospital during 2014 - 2016 were enrolled into the present study. Before study enrollment, written, informed consent for participation was obtained from each patient at the day of hospital admission. Patients with signed agreement were eligible for enrollment if they were $\geq 18$ and $\leq 80$ years old, and preoperative gastric or colorectal cancer patients without distant metastasis. Patients were excluded from this study if they had liver dysfunction, respiratory dysfunction, cardiac dysfunction, renal failure, ongoing infection, history of recent immunosuppressive or immunologic disease (including preoperative chemotherapy and/or radiation therapy), or diabetes mellitus.

\section{Postoperative nutrition protocol}

Subjects were randomly divided into two groups $(\mathrm{n}=8$ in each group) before surgery: 1) group L, who received a peripheral PN (PPN) solution containing 7.5\% glucose and 30\% amino acid (BFLUID Injection; Ohtsuka Pharmaceutical Factory Inc., Tokushima, Japan) and 20\% lipid emulsion (Intralipos Injection 20\%; Ohtsuka Pharmaceutical Factory Inc.); and 2) group LC, who received the same PPN solution plus carnitine (L-CARTIN FF Injection; Otsuka Pharmaceutical, Tokyo, Japan) intravenously. PPN was performed from postoperative day (POD) 1 to POD4 without oral nutrition. Peripheral intravenous infusion of $4.3 \%$ or $10 \%$ glucose with electrolyte solutions was added if necessary to supply water and electrolytes in both groups. Oral water intake was started from POD2 and oral nutritional intake was started from POD4 based on the clinical experience of the doctors in charge of each patient, as well as the condition of each patient.

\section{Clinical and laboratory assessments}

Clinical factors, such as age, sex, preoperative body mass index (BMI), postoperative complications, and postoperative nutritional supplementation (i.e., the amount of calories, amino acids, and lipids administered), were compared between groups L and LC. Blood samples were obtained during the preoperative period, as well as on POD1, POD3, and POD4 for laboratory assessments including hematological data and blood chemistry (i.e., white blood cell count (WBC), total lymphocyte count (TLC), red blood cell count, hemoglobin concentration, hema- tocrit, platelet count (Plt), serum albumin, aspartate aminotransferase (AST), alanine aminotransferase (ALT), cholinesterase (ChE), alkaline phosphatase, total bilirubin (T-bil), blood urea nitrogen (BUN), creatinine (Cre), triglycerides (TG), total cholesterol (TC), transthyretin, retinol-binding protein, fasting plasma glucose (FPG), and fasting plasma insulin (FPI)). Serum concentrations of total carnitine, consisting of both free carnitine and acyl-carnitine, were also determined during the preoperative period and on POD1, POD3, and POD7. Creactive protein (CRP) was determined on POD3 and POD7. Insulin resistance was evaluated using the homeostasis model assessment of insulin resistance (HOMA-IR), calculated as follows [18]: HOMA-IR $=(\mathrm{FPG} \times \mathrm{FPI}) / 405$. Urine samples were collected on POD3 and POD7 for measurement of urinary urea nitrogen to calculate the nitrogen balance.

\section{Statistical analysis}

Statistical analyses were performed using SPSS Statistics version 22 (IBM Japan, Tokyo, Japan). Categorical variables are presented as frequencies. All continuous variables were checked for normal distribution using the Shapiro-Wilk test, and data are expressed as the mean $\pm \mathrm{SD}$ for variables with a normal distribution, and as median values with the interquartile range (IQR) for variables that were not normally distributed. The significance of differences between groups was evaluated using Student's $t$-test or the Mann-Whitney U-test for continuous variables, and Fisher's exact test for categorical variables. Repeated-measures analysis of variance (ANOVA) with Bonferroni's post hoc correction was used for continuous variables with normal distribution to analyze group and postoperative time point differences, whereas the Friedman test and Bonferroni test were used for variables that were not normally distributed. Statistical significance was set at two-tailed $\mathrm{P}<0.05$.

\section{Results}

\section{Pre- and perioperative clinical and laboratory features}

In all, 16 patients were enrolled in the study (eight each in groups L and LC). All the patients in both groups received $100 \mathrm{~mL}$ intravenous 20\% lipid emulsion daily from POD1 to POD4, and the mean dose of carnitine administered intravenously to group LC patients was $72.6 \pm 37.3 \mathrm{mg} / \mathrm{kg} /$ day.

The characteristics of the patients in groups L and LC are given in Table 1. Age, gender, tumor location, cancer stage, and surgical procedure were comparable between the two groups. Preoperative BMI was higher in group LC than in group L (P $<0.05)$; however, postoperarive BMI at POD7 was comparable between the two groups. During postoperative PPN from POD1 to POD4, intravenous administration of both total energy and amino acids was equivalent between the two groups.

Most laboratory parameters of preoperative period, including nitrogen balance, were comparable between the two groups; however, TLC and ChE were significantly higher in group LC than in group $\mathrm{L}(\mathrm{P}<0.05)$. Insulin resistance, as determined by 
Table 1. Patient Characteristics Before and After Surgery

\begin{tabular}{|c|c|c|c|}
\hline & Group L $(n=8)$ & Group LC $(n=8)$ & P-value \\
\hline Age (years) & $69.3 \pm 10.0$ & $66.8 \pm 9.2$ & 0.611 \\
\hline Gender & & & 0.315 \\
\hline Male & $3(37.5)$ & $6(75.0)$ & \\
\hline Female & $5(62.5)$ & $2(25.0)$ & \\
\hline \multicolumn{4}{|l|}{ BMI $\left(\mathrm{kg} / \mathrm{m}^{2}\right)$} \\
\hline Preoperative & $21.4 \pm 3.0$ & $24.8 \pm 2.9$ & 0.038 \\
\hline POD7 & $20.8 \pm 3.4$ & $24.3 \pm 2.5$ & 0.073 \\
\hline Tumor location & & & 0.767 \\
\hline Gastric & $1(12.5)$ & $1(12.5)$ & \\
\hline Colorectal & $7(87.5)$ & $7(87.5)$ & \\
\hline Tumor stage & & & 0.282 \\
\hline I & $4(50.0)$ & $7(87.5)$ & \\
\hline II & $1(12.5)$ & $0(0)$ & \\
\hline III & $3(37.5)$ & $1(12.5)$ & \\
\hline Surgical procedure & & & 0.696 \\
\hline Open & $3(37.5)$ & $3(37.5)$ & \\
\hline Laparoscopic & $5(62.5)$ & $5(62.5)$ & \\
\hline \multicolumn{4}{|l|}{ Intravenous administration } \\
\hline Total energy (kcal/kg/day) & $19.0 \pm 3.2$ & $16.2 \pm 3.0$ & 0.092 \\
\hline Amino acids (g/kg/day) & $1.1 \pm 0.2$ & $0.9 \pm 0.2$ & 0.106 \\
\hline
\end{tabular}

Data are given as the mean \pm SD or as $n(\%)$. Group L: peripheral parenteral nutrition (PPN); Group LC: intravenous carnitine in addition to PPN; BMI: body mass index.

HOMA-IR, was comparable between the two groups. Preoperative serum total carnitine concentrations were comparable between the two groups: concentrations of both free and acyl carnitine were comparable between the two groups (Table 2).

\section{Postoperative changes in laboratory parameters}

Postoperative changes in laboratory parameters were comparable between the two groups (Table 3). There were some withingroup variations in $\mathrm{WBC}$, Plt, AST, T-bil, BUN, TG, and TC, but critically abnormal values were not observed. Serum concentrations of both total and free carnitine were significantly higher in group LC than in group L on POD3 and POD7, and acyl carnitine was higher in group LC than in group L on POD3.

Postoperative CRP concentrations were comparable in the two groups on both POD3 and POD7 (Table 3); however, examining changes in CRP from POD3 to POD7 revealed a significantly greater decrease in CRP levels over this period in group LC than in group $\mathrm{L}(\mathrm{P}=0.011$; Fig. 1$)$.

\section{Postoperative complications and mortality}

The frequency of postoperative complications (i.e., total complications, infectious complications, and/or mechanical com- plications) was comparable between the two groups (Table 4), and no patients died during the study. As infectious complication, one case of intra-abdominal abscess in group L, and one case of enteritis in group LC, were observed, respectively. As mechanical complication, while one case of pleural effusion accompanied with atelectasis in group L was observed, one case of anastomotic leakage and another case of chyle ascites were observed in group LC. The frequency of both infectious and mechanical complications was also comparable between two groups. All participants made a smooth recovery and were discharged from hospital.

\section{Discussion}

Because lipid emulsions are one of the essential constituents of PN [19], they have been used in various clinical conditions, including after surgery and in critically ill patients. Since the creation by Arvid Wretlind of the traditional soybean oil (n-6 polyunsaturated fatty acid (PUFA))-based safe lipid emulsion, different types of lipid emulsions have been developed, including those based on medium-chain triglycerides (MCT), olive oil (n-9 monounsaturated fatty acid (MUFA)), and fish oil (n-3 PUFA) [20].

Because the soybean oil (n-6 PUFA)-based lipid emulsion may induce increased oxidative stress and systemic inflamma- 
Table 2. Preoperative Laboratory Characteristics

\begin{tabular}{|c|c|c|c|}
\hline & Group L $(n=8)$ & Group LC $(n=8)$ & P-value \\
\hline $\mathrm{WBC}(/ \mu \mathrm{L})$ & $5,153 \pm 1,661$ & $6,263 \pm 1,908$ & 0.235 \\
\hline $\operatorname{TLC}(/ \mu \mathrm{L})$ & $1,560(1,428-1,783)$ & $1,838(1,620-2,311)$ & 0.014 \\
\hline $\mathrm{RBC}\left(\times 10^{4} / \mu \mathrm{L}\right)$ & $402 \pm 63$ & $446 \pm 35$ & 0.108 \\
\hline $\mathrm{Hb}(\mathrm{g} / \mathrm{dL})$ & $12.0 \pm 2.7$ & $13.8 \pm 1.1$ & 0.096 \\
\hline $\operatorname{Hct}(\%)$ & $36.1 \pm 6.5$ & $40.9 \pm 3.1$ & 0.780 \\
\hline $\operatorname{Plt}\left(\times 10^{4} / \mu \mathrm{L}\right)$ & $22.6 \pm 4.1$ & $27.2 \pm 9.0$ & 0.215 \\
\hline Alb (g/dL) & $4.1 \pm 0.4$ & $4.2 \pm 0.3$ & 0.459 \\
\hline TTR (mg/dL) & $30.1 \pm 2.6$ & $26.8 \pm 8.0$ & 0.325 \\
\hline $\mathrm{RBP}(\mathrm{mg} / \mathrm{dL})$ & $5.1 \pm 0.8$ & $4.9 \pm 1.5$ & 0.702 \\
\hline AST (U/L) & $21.8 \pm 5.0$ & $21.1 \pm 4.8$ & 0.763 \\
\hline $\operatorname{ALT}(\mathrm{U} / \mathrm{L})$ & $17.3 \pm 4.4$ & $19.4 \pm 5.2$ & 0.417 \\
\hline $\mathrm{ChE}(\mathrm{U} / \mathrm{L})$ & $264.6 \pm 57.0$ & $333.4 \pm 62.4$ & 0.037 \\
\hline $\operatorname{ALP}(\mathrm{U} / \mathrm{L})$ & $232.4 \pm 38.7$ & $254.8 \pm 84.5$ & 0.507 \\
\hline T-bil (mg/dL) & $0.8(0.7-0.9)$ & $0.6(0.4-0.8)$ & 0.130 \\
\hline BUN (mg/dL) & $14.5 \pm 3.8$ & $16.1 \pm 3.4$ & 0.369 \\
\hline Cre $(\mathrm{mg} / \mathrm{dL})$ & $0.6 \pm 0.2$ & $0.8 \pm 0.2$ & 0.179 \\
\hline TG (mg/dL) & $126.2 \pm 71.1$ & $104.1 \pm 35.4$ & 0.483 \\
\hline $\mathrm{TC}(\mathrm{mg} / \mathrm{dL})$ & $190.2 \pm 22.8$ & $186.1 \pm 18.7$ & 0.733 \\
\hline HOMA-IR & $0.9(0.8-2.4)$ & $1.8(1.1-2.1)$ & 0.613 \\
\hline Total carnitine $(\mu \mathrm{mol} / \mathrm{L})$ & $56.0 \pm 13.0$ & $60.0 \pm 7.6$ & 0.470 \\
\hline Free carnitine $(\mu \mathrm{mol} / \mathrm{L})$ & $44.8 \pm 11.1$ & $50.2 \pm 8.0$ & 0.291 \\
\hline Acyl carnitine $(\mu \mathrm{mol} / \mathrm{L})$ & $11.3 \pm 3.7$ & $9.8 \pm 3.0$ & 0.410 \\
\hline
\end{tabular}

Data are given as the mean \pm SD or as the median (interquartile range). Group L: peripheral parenteral nutrition (PPN); Group LC: intravenous carnitine in addition to PPN; WBC: white blood cell count; TLC: total lymphocyte count; RBC: red blood cell count; Hb: hemoglobin; Hct: hematocrit; Plt: platelet count; Alb: serum albumin; TTR: transthyretin; RBP: retinol-binding protein; AST: aspartate aminotransferase; ALT: alanine aminotransferase; ChE: cholinesterase; ALP: alkaline phosphatase; T-bil: total bilirubin; BUN: blood urea nitrogen; Cre: creatinine; TG: triglycerides; TC: total cholesterol; HOMA-IR: homeostasis model assessment of insulin resistance.

tion via the production of pro-inflammatory prostanoids and leukotrienes [21, 22], it may be associated with worse clinical outcomes under the stressed state [23]. Compared with n-6 PUFA-based lipid emulsions, MCT-, n-9 MUFA-, or n-3 PUFA-based lipid emulsions have less inflammatory activity [24]. Therefore, it has been recommended that $100 \%$ soybean oil (n-6 PUFA)-based emulsions are not administered to critically ill patients [10]. In addition, a recent meta-analysis showed that alternative oil-based lipid emulsions may be associated with clinically important reductions in mortality [24].

In surgical patients, compared with fish oil (n-3 PUFA)based lipid emulsions [25], soybean oil (n-6 PUFA)-based lipid emulsions have been shown to be less beneficial in improving immunologic function and inflammatory responses $[26,27]$, in decreasing the length of hospitalization [28], and in reducing the risk of clinical complications [27]. Therefore, it is not recommended that soybean oil (n-6 PUFA)-based emulsions are administered to surgical patients. In the present study, we used a soybean oil (n-6 PUFA)-based emulsion because it is the only type of lipid emulsion permitted for use in clinical situations in Japan.
A clinical comparison was made to elucidate the efficacy of the addition of carnitine to PPN with lipid emulsion in enhancing LCFA $\beta$-oxidation, improving nutritional status, and decreasing insulin resistance. As summarized in Table 3, the results did not show an effect of the addition of carnitine in improving nutritional status or decreasing insulin resistance. There were no significant differences in postoperative changes in serum albumin, transthyretin, and retinol-binding protein, in the nitrogen balance, or in the postoperative changes in $\mathrm{HO}-$ MA-R between groups L and LC. One possible reason why we did not detect any positive effects of carnitine administration on improvements in nutrition and/or insulin resistance is the relatively short duration of PPN administration. The 3 - 4 days of PPN support in the present study do not appear to be long enough for the effects of carnitine on nutrition and insulin resistance to become apparent.

In the present study, preoperative BMI, TLC and ChE were higher in group LC than in group $\mathrm{L}(\mathrm{P}<0.05)$. We suppose that higher value of these parameters in group LC reflected better perioperative nutritional status of group LC compared with 


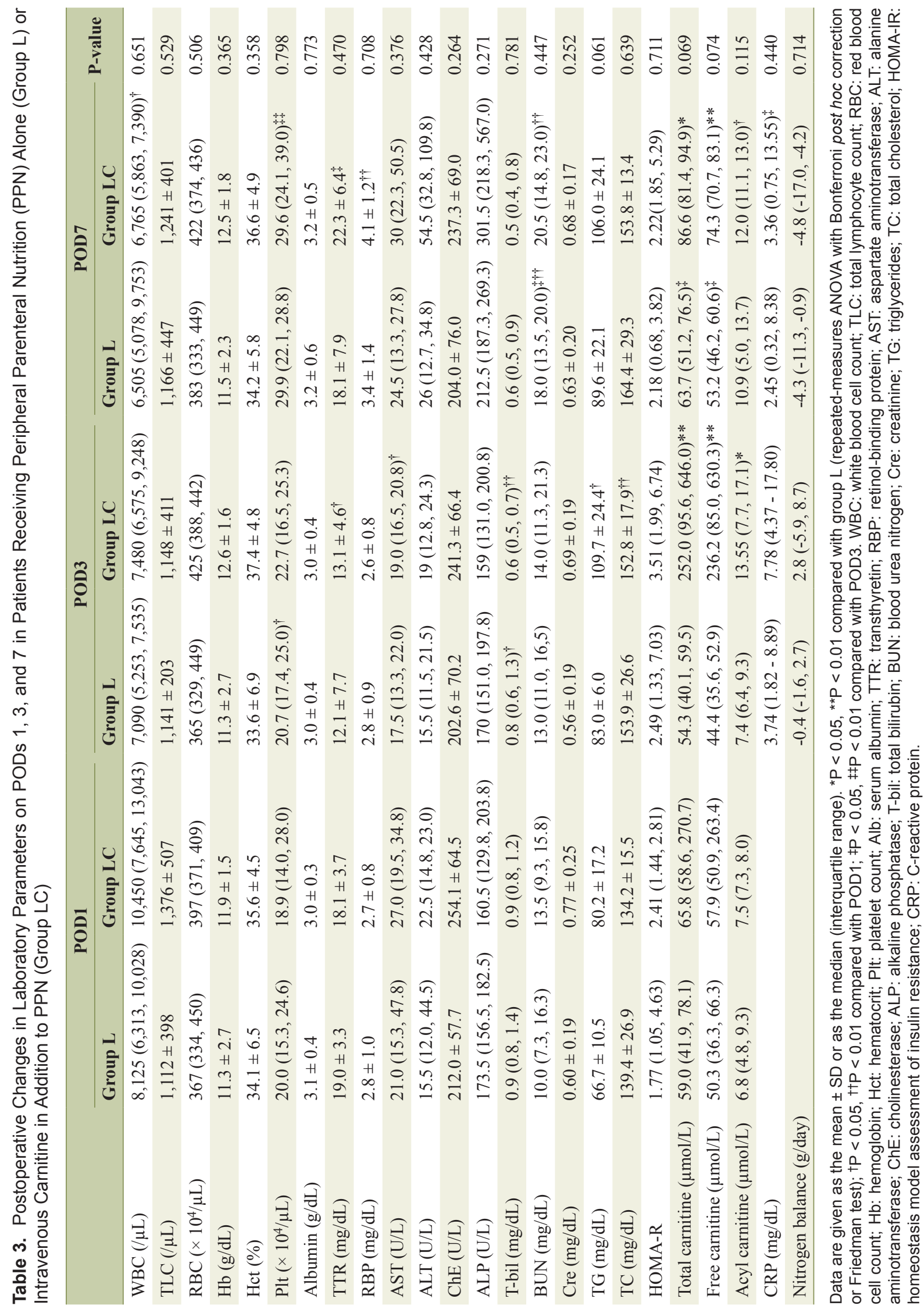




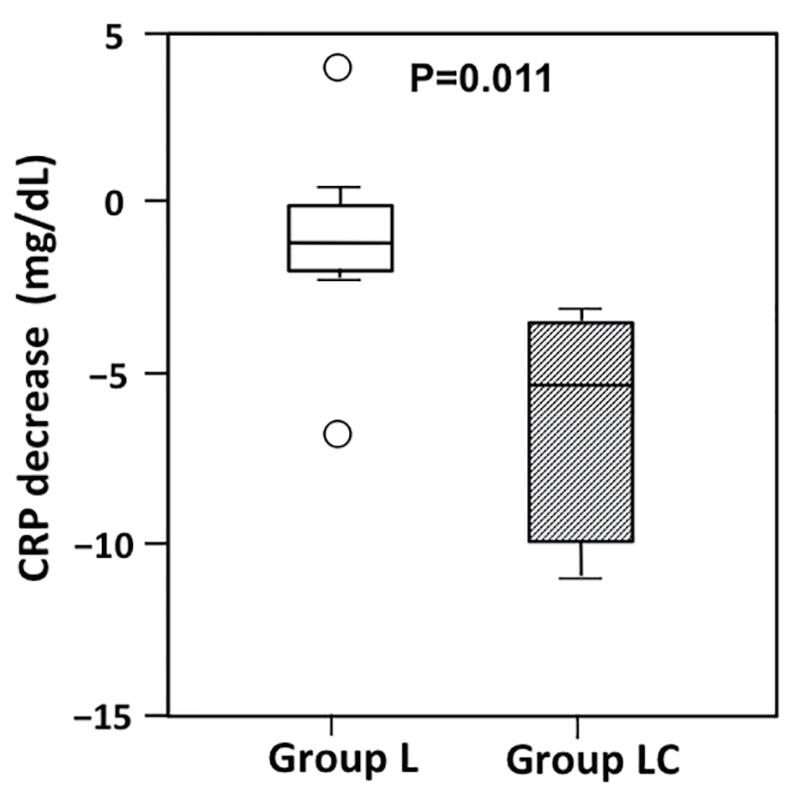

Figure 1. Changes in C-reactive protein (CRP) from postoperative day (POD) 3 to POD7 in patients receiving peripheral parenteral nutrition (PPN) alone (group L) or intravenous carnitine in addition to PPN (group LC). The decrease in CRP from POD3 to POD7 was significantly greater in group $L C$ than in Group $L(P=0.011)$. The boxes show the interquartile range, with the median value indicated by the horizontal line; whiskers show the range. Circles show outliers.

group L because BMI, TLC and/or ChE are the parameters that will be often used for nutritional assessment, and postoperative changes of these parameters were comparable between the two groups in this sutdy.

Conversely, the decrease in CRP from POD3 to POD7 was significantly greater in group LC than in group L (Fig. 1). The greater decrease in CRP in group LC may reflect enhanced LCFA (i.e. n-6 PUFA) $\beta$-oxidation: because n-6 PUFAs, which induce oxidative stress and systemic inflammation, are responsible for CRP production, an increase in n-6 PUFA $\beta$-oxidation following carnitine administration will result in decreased CRP production. Other laboratory parameters (i.e., AST, ALT, BUN, and Cre) were comparable between the two groups, as were postoperative complications, indicating that carnitine administration in addition to PPN was safe in the present study. In addition, because the results of the present study revealed higher concentrations of both total and free carnitine in group LC compared with group L on both POD3 and POD7, the effects of carnitine administration may persist during the postadministration period, and such a "carry-over" effect may also contribute to the significant decrease in CRP seen in group LC.

\section{Limitations}

The main limitation of the present study is the small number of patients enrolled: some patients declined to participate in the study and others were excluded because of preoperative complications as per the exclusion criteria. The short PPN period of 3 - 4 days in the present study may also have been insuf-
Table 4. Postoperative Complications in Patients Receiving Peripheral Parenteral Nutrition (PPN) Alone (Group L) or Intravenous Carnitine in Addition to PPN (Group LC)

\begin{tabular}{|c|c|c|c|}
\hline & Group L & Group LC & P-value \\
\hline Postoperative complications & & & 0.715 \\
\hline Yes & $2(25.0)$ & $2(25.0))$ & \\
\hline No & $6(75.0)$ & $6(75.0)$ & \\
\hline \multicolumn{4}{|l|}{ Infectious complications } \\
\hline Yes & $1(12.5)$ & $1(12.5)$ & 0.767 \\
\hline No & $7(87.5)$ & $7(87.5)$ & \\
\hline Mechanical complications & & & 0.500 \\
\hline Yes & $1(12.5)$ & $2(25.0)$ & \\
\hline No & $7(87.5)$ & $6(75.0)$ & \\
\hline
\end{tabular}

Data show the number of patients in each group, with percentages in parentheses.

ficient to demonstrate the positive effects of carnitine administration on improvements in nutrition and/or insulin resistance.

\section{Conclusion}

The results of the present study show that intravenous carnitine administration in addition to PN with lipid emulsion is safe and may be beneficial for recovery from postoperative inflammatory reactions. We hope that the findings of the present study provide a cornerstone for future clinical research into the efficacy of carnitine administration.

\section{Acknowledgments}

The authors thank Dr Yasunobu Matsuda at Niigata University Graduate School of Health Sciences and Dr Yoshinori Kamiya at Niigata University Graduate School of Medical and Dental Sciences for their excellent advises regarding data analysis.

\section{Conflicts of Interest}

The authors declare that there are no conflicts of interest.

\section{Grant Support}

This work was supported financially by Grants-in-Aid for Science Research from the Ministry of Education, Science, Sports and Culture, Japan (Project no. 15K10047).

\section{Author Contributions}

Kazuki Moro, Toshifumi Wakai and Yu Koyama designed the research. Shin-ichi Kosugi, Takaaki Hanyu, Hiroshi Ichikawa, 
Masayuki Nagahashi and Junko Tsuchida conducted the research. Masato Nakano, Masato Nakajima, Jun Sakata, and Kohei Miura collected the data. Mayuko Ikarashi, Yoshifumi Shimada, Takashi Kobayashi, Hitoshi Kameyama and Yu Koyama analyzed the data. Yu Koyama summarized the data and wrote the manuscript and has the primary responsibility for the final content. All authors read, checked, and approved the final manuscript.

\section{References}

1. O'Donnell J, Finer NN, Rich W, Barshop BA, Barrington KJ. Role of L-carnitine in apnea of prematurity: a randomized, controlled trial. Pediatrics. 2002;109(4):622-626.

2. Rebouche CJ. Carnitine function and requirements during the life cycle. FASEB J. 1992;6(15):3379-3386.

3. Ferrari R, Merli E, Cicchitelli G, Mele D, Fucili A, Ceconi C. Therapeutic effects of L-carnitine and propionyl-Lcarnitine on cardiovascular diseases: a review. Ann N Y Acad Sci. 2004;1033:79-91.

4. Kepka A, Chojnowska S, Okungbowa OE, Zwierz K. The role of carnitine in the perinatal period. Dev Period Med. 2014;18(4):417-425.

5. Winther B, Jackson D, Mulroy C, MacKay M. Evaluation of serum carnitine levels for pediatric patients receiving carnitine-free and carnitine-supplemented parenteral nutrition. Hosp Pharm. 2014;49(6):549-553.

6. Boullata JI, Gilbert K, Sacks G, Labossiere RJ, Crill C, Goday P, Kumpf VJ, et al. A.S.P.E.N. clinical guidelines: parenteral nutrition ordering, order review, compounding, labeling, and dispensing. JPEN J Parenter Enteral Nutr. 2014;38(3):334-377.

7. Demirer S, Sapmaz A, Karaca AS, Kepenekci I, Aydintug S, Balci D, Sonyurek P, et al. Effects of postoperative parenteral nutrition with different lipid emulsions in patients undergoing major abdominal surgery. Ann Surg Treat Res. 2016;91(6):309-315.

8. Calder PC. Immunonutrition in surgical and critically ill patients. Br J Nutr. 2007;98(Suppl 1):S133-139.

9. Rayyan M, Devlieger H, Jochum F, Allegaert K. Shortterm use of parenteral nutrition with a lipid emulsion containing a mixture of soybean oil, olive oil, medium-chain triglycerides, and fish oil: a randomized double-blind study in preterm infants. JPEN J Parenter Enteral Nutr. 2012;36(1 Suppl):81S-94S.

10. Adolph M, Heller AR, Koch T, Koletzko B, Kreymann $\mathrm{KG}$, Krohn K, Pscheidl E, et al. Working group for developing the guidelines for parenteral nutrition of The German Association for Nutritional Medicine. Lipid emulsions - Guidelines on Parenteral Nutrition, Chapter 6. Ger Med Sci. 2009;7:Doc22.

11. Bonner CM, DeBrie KL, Hug G, Landrigan E, Taylor BJ. Effects of parenteral L-carnitine supplementation on fat metabolism and nutrition in premature neonates. J Pediatr. 1995;126(2):287-292.

12. Schmidt-Sommerfeld E, Penn D, Wolf H. Carnitine deficiency in premature infants receiving total parenteral nutrition: effect of L-carnitine supplementation. J Pediatr. 1983;102(6):931-935.
13. Coran AG, Drongowski RA, Baker PJ. The metabolic effects of oral L-carnitine administration in infants receiving total parenteral nutrition with fat. J Pediatr Surg. 1985;20(6):758-764.

14. Cairns PA, Stalker DJ. Carnitine supplementation of parenterally fed neonates. Cochrane Database Syst Rev. 2000;4:CD000950.

15. Clark RH, Chace DH, Spitzer AR. Impact of 1-carnitine supplementation on metabolic profiles in premature infants. J Perinatol. 2017;37(5):566-571.

16. Seong SH, Cho SC, Park Y, Cha YS. L-carnitine-supplemented parenteral nutrition improves fat metabolism but fails to support compensatory growth in premature Korean infants. Nutr Res. 2010;30(4):233-239.

17. Crill CM, Storm MC, Christensen ML, Hankins CT, Bruce Jenkins M, Helms RA. Carnitine supplementation in premature neonates: effect on plasma and red blood cell total carnitine concentrations, nutrition parameters and morbidity. Clin Nutr. 2006;25(6):886-896.

18. Muneyuki T, Nakajima K, Aoki A, Yoshida M, Fuchigami $\mathrm{H}$, Munakata $\mathrm{H}$, Ishikawa $\mathrm{SE}$, et al. Latent associations of low serum amylase with decreased plasma insulin levels and insulin resistance in asymptomatic middle-aged adults. Cardiovasc Diabetol. 2012;11:80.

19. Vanek VW, Seidner DL, Allen P, Bistrian B, Collier S, Gura K, Miles JM, et al. A.S.P.E.N. position paper: Clinical role for alternative intravenous fat emulsions. Nutr Clin Pract. 2012;27(2):150-192.

20. Nordenstrom J. Surgical contributions to Intralipid development. Clin Nutr. 2003;22(Suppl 2):S13-15.

21. Wanten GJ, Calder PC. Immune modulation by parenteral lipid emulsions. Am J Clin Nutr. 2007;85(5):1171-1184.

22. Furukawa K, Yamamori H, Takagi K, Hayashi N, Suzuki R, Nakajima N, Tashiro T. Influences of soybean oil emulsion on stress response and cell-mediated immune function in moderately or severely stressed patients. Nutrition. 2002;18(3):235-240.

23. Heyland DK, MacDonald S, Keefe L, Drover JW. Total parenteral nutrition in the critically ill patient: a metaanalysis. JAMA. 1998;280(23):2013-2019.

24. Manzanares W, Dhaliwal R, Jurewitsch B, Stapleton RD, Jeejeebhoy KN, Heyland DK. Alternative lipid emulsions in the critically ill: a systematic review of the evidence. Intensive Care Med. 2013;39(10):1683-1694.

25. Klek S. Omega-3 Fatty Acids in Modern Parenteral Nutrition: A Review of the Current Evidence. J Clin Med. 2016;5(3):34.

26. Wang J, Yu JC, Kang WM, Ma ZQ. Superiority of a fish oil-enriched emulsion to medium-chain triacylglycerols/long-chain triacylglycerols in gastrointestinal surgery patients: a randomized clinical trial. Nutrition. 2012;28(6):623-629.

27. Han YY, Lai SL, Ko WJ, Chou CH, Lai HS. Effects of fish oil on inflammatory modulation in surgical intensive care unit patients. Nutr Clin Pract. 2012;27(1):91-98.

28. Zhu X, Wu Y, Qiu Y, Jiang C, Ding Y. Effects of omega-3 fish oil lipid emulsion combined with parenteral nutrition on patients undergoing liver transplantation. JPEN J Parenter Enteral Nutr. 2013;37(1):68-74. 